\title{
Deposition of bio-mimicking graphene sheets with lotus leaf-like and cell-like structures on the nickel substrate
}

\author{
YANG Juan, YAN XingBin*, WANG Ying, LUO BaoMin, WANG LiPing \& XUE QunJi \\ State Key Laboratory of Solid Lubrication, Lanzhou Institute of Chemical Physics, Chinese Academy of Sciences, Lanzhou 730000, China
}

Received March 6, 2012; accepted May 3, 2012; published online June 8, 2012

\begin{abstract}
Bio-mimicking graphene films, deposited on textured nickel substrates, were synthesized by the following method: replicating the surface textures of the lotus leaf by polymer duplication, fabricating textured nickel substrates by electroplating on the polymer coated with a Au film, preparing bio-mimicking graphene oxide films on the nickel substrates by vacuum filtration, and electrochemical reduction. By controlling the vacuum filtration, this replica method can not only replicate the lotus leaf structure by a graphene film, but also can achieve a novel cell-like graphene film.
\end{abstract}

bio-mimicking, graphene film, lotus leaf-like structure, cell-like structure

Citation: Yang J, Yan X B, Wang Y, et al. Deposition of bio-mimicking graphene sheets with lotus leaf-like and cell-like structures on the nickel substrate. Chin Sci Bull, 2012, 57: 3036-3039, doi: 10.1007/s11434-012-5310-0

Since graphene was first isolated in 2004 with the help of Scotch Tape, researchers have excitedly turned to the material to discover its potential applications, which include nanoelectronics, sensors, batteries, supercapacitors, hydrogen storage and nanocomposites [1-5]. Based on the excellent flexibility and mechanical stiffness, graphene oxide (GO) and graphene sheets can indeed be assembled into paper-like materials through flow-directed assembly by vacuum filtration of their colloidal dispersions [6,7]. Also, employing their stretchable physical property, GO and graphene films can be transferred to an arbitrary substrate [8].

Biomimic micro textures, owing to their intrinsic geometric microstructure, bring about many unique properties [9-14]. It is well known that the lotus leaf structure, which includes micro-bumps and a thin wax film on the surface, has attracted much interest with its self-cleaning function and low hysteresis ability to carry effortlessly the contaminations attached to the leaf when the surface is slightly tilted. Accordingly, there has been much effort to design and synthesize multifunctional artificial materials by mimicking the surface of the lotus leaf [15-19]. It is expected that, if gra-

*Corresponding author (email: xbyan@licp.cas.cn) phene sheets were assembled into bio-mimicking lotus leaf surfaces, it would stimulate some novel functional properties for various potential applications.

In this study, a preparation of the bio-mimicking graphene films with lotus leaf-like and cell-like structures on nickel substrates was first reported. A simple combination of duplication-electroplating resulted in fully duplicated surface micro textures of lotus leaf on nickel substrates. A controllable vacuum filtration realized the textured GO films on the bio-mimicking nickel substrates. Owing to the excellent flexibility and ultrathin structure of GO and graphene sheets, not only the morphology of bio-mimicking nickel substrates can be retained, also the original isolated micro-bumps on the surface can be linked with graphene sheets through prolonging the titration time during the vacuum filtration.

\section{Experimental}

A fresh lotus leaf was placed in a glass dish. Polydimethylsiloxane (PDMS) was poured over the leaf, held for $2 \mathrm{~h}$ at room temperature (RT) and then cured in a drying oven at $60^{\circ} \mathrm{C}$ for $10 \mathrm{~h}$, to replicate the surface micro textures of the 
lotus leaf. The textured PDMS film was gently peeled off from the lotus leaf. After that, a gold coating with an approximate thickness of $100 \mathrm{~nm}$ was sputtered on the textured PDMS and then a nickel layer (the thickness was above $100 \mu \mathrm{m}$ ) was electrodeposited on the surface of the gold coating. The PDMS mold was peeled off from the textured nickel layer, to form a freestanding nickel slice. GO was prepared from natural graphite [20-22] (See the Supporting Information for details). A homogeneous GO aqueous suspension $(0.5 \mathrm{mg} / \mathrm{mL})$ was obtained with the aid of sonication using a high-power $(800 \mathrm{~W})$ ultrasonic pole at $0^{\circ} \mathrm{C}$ for $1 \mathrm{~h}$, and then the suspension was centrifuged at $3000 \mathrm{r} / \mathrm{min}$ for $30 \mathrm{~min}$. The resulting suspension was placed in a funnel and dropwise added ( $1 \mathrm{drop} / \mathrm{min})$ on the textured nickel slice $(1 \mathrm{~cm} \times 1 \mathrm{~cm})$, which was fixed on the center of a Millipore filter $(50 \mathrm{~mm}$ in diameter and $0.45 \mu \mathrm{m}$ in pore size). The vacuum filtration experiment was carried out for different durations ( 2 and $4 \mathrm{~d}$ ) to prepare bio-mimicking GO films with different surface textures. Finally, the textured GO films were electrochemically reduced in the $0.1 \mathrm{~mol} / \mathrm{L}$ $\mathrm{KCl}$ solution using an Autolab Electrochemical Working Station. The textured GO and graphene films were characterized by field emission scanning electron microscope (FE-SEM, JSM-6701F) and Raman spectroscopy (JY-HR800, the excitation wavelength at $532 \mathrm{~nm}$ ). The electrical conductivity was measured by a standard four-probe method.

\section{Results and discussion}

As shown in Figure 1, the success of the technique in the process flow sheet to create the graphene film of biomimicking lotus leaf requires four key steps. Firstly, a PDMS film is used to replicate the surface micro textures of the lotus leaf to obtain a negative impression of the bio- mimicking micro textures. Secondly, a nickel layer is electrodeposited on top of the textured PDMS film to obtain the positive replica of the lotus leaf after removing the textured polymer film. In this step, since the PDMS is an insulating material, a thin gold coating should be sputtered on the surface of the textured PDMS in advance, to aid the subsequent electroplating of nickel. Thirdly, a GO film is placed on top of the textured nickel substrate by vacuum filtration. In this step, through adjusting the titration time of the GO suspension during the filtration, two different morphologies can be obtained. Finally, the textured GO films are electrochemically reduced into textured graphene films at RT.

SEM images revealed the surface textures of the lotus leaf and its replicas. Figure 2(a) demonstrates the arrayed part of the bumps on the textured nickel surface as well as the one-dimensional order of the microstructure similar to that of the SEM image of the lotus leaf (shown in the inset). Figure 2(b) presents a typical low-magnification SEM image of the textured GO film when the copy of the structure

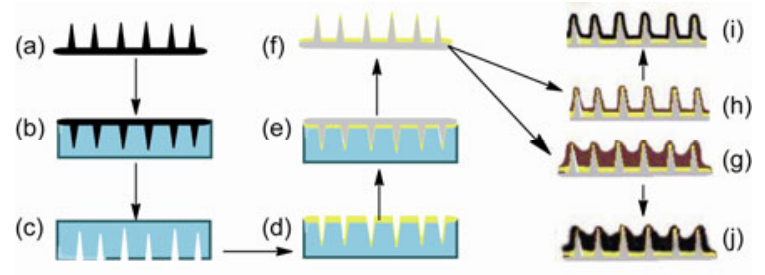

Figure 1 (Color online) The process of creating the graphene films with bio-mimicking textures: (a) the original lotus leaf; (b) replicating the surface micro textures of the lotus leaf with PDMS; (c) the PDMS film with a negative impression of the bio-mimicking textures; (d) sputtering gold on the textured PDMS film; (e) electrodepositing nickel layer on the surface of the textured PDMS film; (f) the nickel slice with the bio-mimicking textures; (g) and (i) the GO and graphene films with lotus leaf-like structures prepared by vacuum filtration followed by electrochemical reduction; (h) and (j) the GO and graphene films with cell-like structures prepared by vacuum filtration followed by electrochemical reduction.
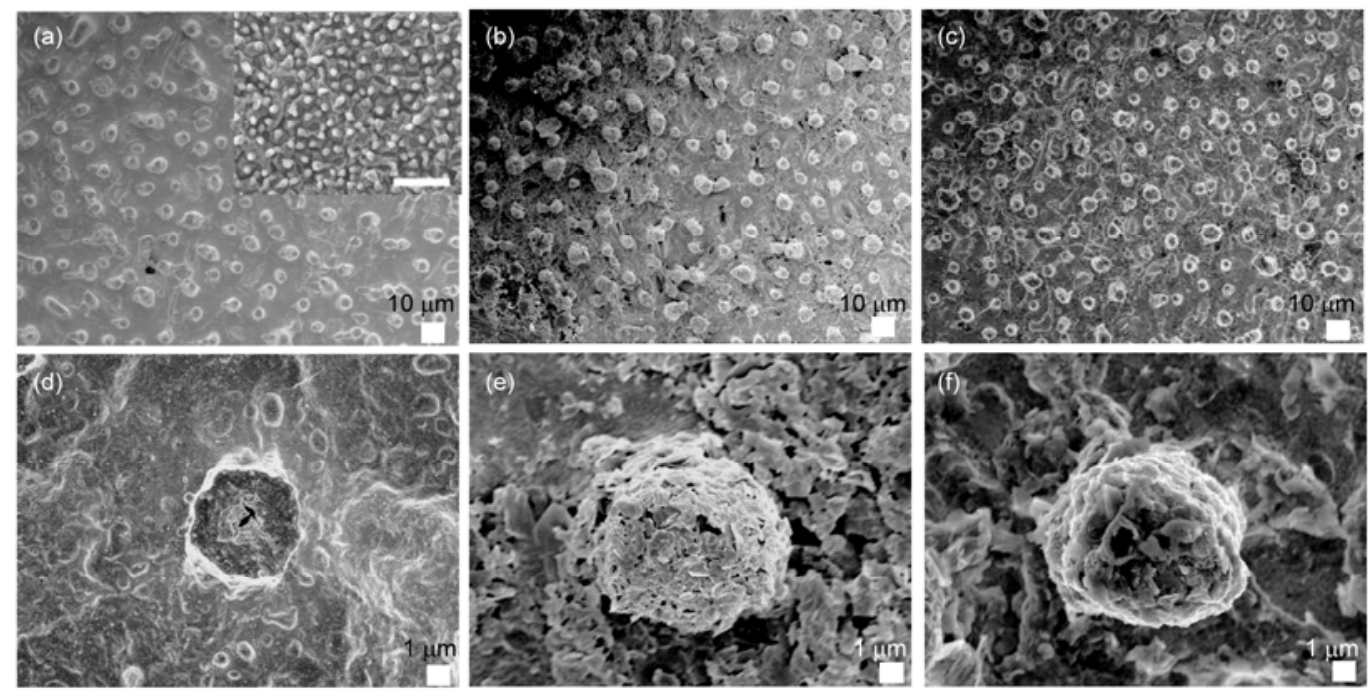

Figure 2 SEM images of top view of the textured nickel slice (a) and (d); the textured GO film with lotus leaf-like structures (b) and (e); and the textured graphene film with lotus leaf-like structures (c) and (f). The inset in (a) is the typical SEM image of the lotus leaf and the scale is $50 \mu \mathrm{m}$. 
of the lotus leaf was completed. The textured GO film was then electrochemically reduced into the graphene film and the corresponding low-magnification SEM image is shown in Figure 2(c). It is clear seen that the surfaces of the as-obtained GO and graphene films were both composed of micro-scale bump arrays, which were in accordance with the surface morphology of the textured nickel slice. The average size the bumps was at about 3-6 $\mu \mathrm{m}$ and the distance between the adjacent bumps was of about 5-20 $\mu \mathrm{m}$. Figure 2(d)-(f) shows the typical SEM images of the individual bumps on nickel, GO and graphene films. Owing to the wrapping of the GO and graphene, the sizes of the bumps on the textured GO and graphene films were larger than that of the bump on the textured nickel slice.

In our vacuum filtration step, through prolonging the titration time of GO suspension, another surface morphology was obtained on the GO film and on the subsequent graphene film. As shown in Figure 3(a) and (c), some two adjacent bumps were linked with GO sheets. Such morphology was also retained after the electrochemical reduction of GO (Figure 3(b) and (d)). In other words, some adjacent bumps were connected with graphene sheets, as a result of the formation of the textured graphene film with cell-like structures.

As shown in Figure 4, the Raman spectra of two textured GO films were identical, which presented two features: D peak at $1358 \mathrm{~cm}^{-1}$ and $\mathrm{G}$ peak $1605 \mathrm{~cm}^{-1}$, i.e. assigned to $\mathrm{sp}^{2}$-hybridized $\mathrm{C}-\mathrm{C}$ bonds. Although the Raman spectra of two textured graphene films also had both $\mathrm{D}$ and $\mathrm{G}$ bands at the similar positions, the intensity ratio of $D / G$ increased obviously in comparison with that of the GO spectra. It agreed well with the graphene materials prepared by hydrazine reduction $[22,23]$, indicating the realization of deoxygenation in electrochemically reduced graphene films. In addition, the result of electrical conductivity measurements showed that the electrical conductivity of the textured graphene films was approximately $15 \mathrm{~S} / \mathrm{cm}$, which was much larger than that of the textured GO films $\left(<10^{-7} \mathrm{~S} / \mathrm{cm}\right)$.

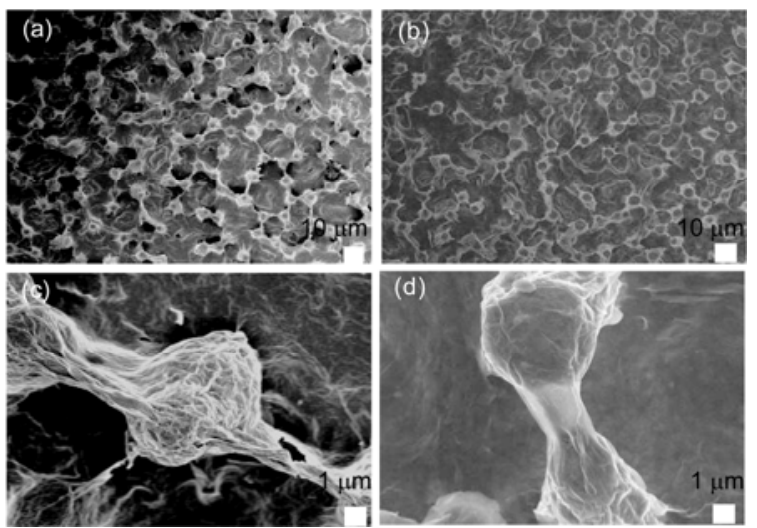

Figure 3 SEM images of top view of the textured GO film with cell-like structures (a) and (c); and the textured graphene film with cell-like structures (b) and (d).

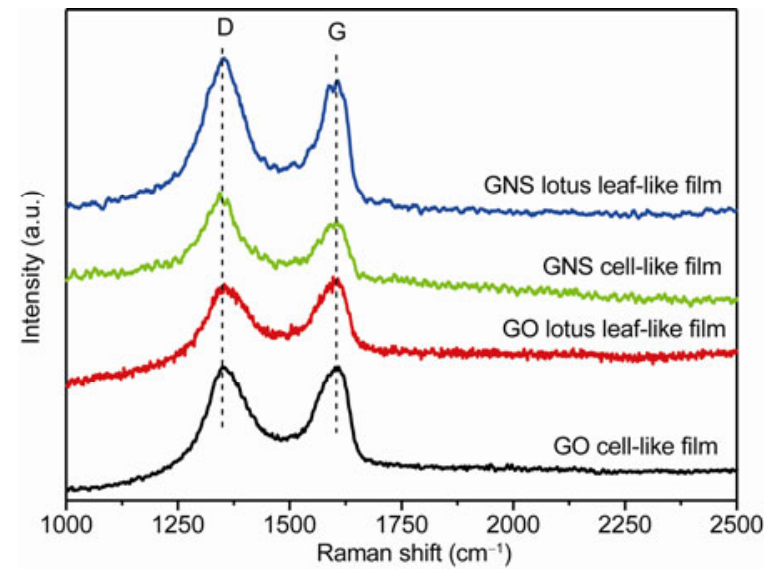

Figure 4 (Color online) Raman spectra of the bio-mimicking GO and graphene films with lotus leaf-like structures and cell-like structures.

X-ray photoelectron spectroscope further revealed that the GO film was deoxygenized by electrochemical reduction (see the Supporting Information for details). Therefore, electrochemical reduction of GO, using applied DC bias by scanning the potential at RT, is in favor of fabricating highly ordered and controllable graphene films on the electrode material [24].

\section{Conclusions}

It is well known that GO sheets have excellent flexibility and mechanical stiffness. In our synthesis, when the GO suspension was dropwise added on the textured nickel slice which was fixed on the Millipore filter, GO sheets were adsorbed onto the textured nickel surface and onto exposed filter surface through flow-directed assembly by vacuum filtration. Because of the relatively high pressure of vacuum filtration (0.08 MPa) and slow droplet rate, GO sheets adsorbed on the micro-scale bumps bent and then wrapped around the bumps under the flow-inductive force. As a result the morphology of the obtained GO film was in accordance with the textured nickel slice. With prolongation of titration time, the flow-inductive force became weaker and weaker. In this situation, the GO sheets freshly absorbed on the bumps, especially some large size GO sheets simultaneously contacted with the adjacent two bumps, failed to bend completely and wrap around the bumps. On the contrary, they merely linked with the adjacent bumps and other parts bent towards the flow direction, as a result of the formation of the cell-like structures. It should be mentioned that, the droplet rate and the total titration time of the GO suspension must be carefully controlled in the replication of the micro-scale bumps from nickel surfaces by using vacuum filtration. With the relatively short titration time of $2 \mathrm{~d}$, the GO film with lotus leaf-like structures was formed; with the relatively long titration time of $4 \mathrm{~d}$, the GO film with cell-like structures was formed. 
The successful preparation of the textured GO and graphene films on nickel slices proved that the flow-directed assembly by vacuum filtration is an efficient approach to replicate the micro-scale bumps on the nickel surface. Furthermore, with the controlled droplet rate and calculated increase in the quantity of the GO suspension, the new cell-like GO and graphene films can be prepared. We believe that, this strategy can be used to prepare the textured GO and graphene films on different substrates with various geometric microstructures. Also, the textured graphene films with bio-mimicking structures are promising materials for various novel applications, such as nanoelectronics, electrodes, capacitors, batteries and culture of electrically excitable cells.

This work was supported by the "Hundred Talants Program" of the Chinese Academy of Sciences and the National Natural Science Foundation of China (51005225 and 51002161).

1 Pang S P, Tsao H N, Feng X L, et al. Patterned graphene electrodes from solution processed graphite oxide films for organic field-effect transistors. Adv Mater, 2009, 21: 3488-3491

2 Su Q, Pang S P, Alijani V, et al. Composites of graphene with large aromatic molecules. Adv Mater, 2009, 21: 3191-3195

3 Wang X, Zhi L J, Tsao N, et al. Transparent carbon films as electrodes in organic solar cells. Angew Chem Int Ed, 2008, 47: 2990-2992

4 Liang Y Y, Wu D Q, Feng X L, et al. Dispersion of graphene sheets in organic solvent supported by ionic interactions. Adv Mater, 2009, 21: $1679-1683$

5 Stoller M D, Park S, Zhu Y W, et al. Graphene-based ultracapacitors. Nano Lett, 2008, 8: 3498-3502

6 Dikin D A, Stankovich S, Zimney E J, et al. Preparation and characterization of graphene oxide paper. Nature, 2007, 448: 457-760

7 Li D, Müller M B, Gilje S, et al. Processable aqueous dispersions of graphene nanosheets. Nat Nanotechnol, 2008, 3: 101-105

8 Kim K S, Zhao Y, Jang H, et al. Large-scale pattern growth of gra- phene films for stretchable transparent electrodes. Nature, 2009, 457: 706-710

9 Feng L, Zhang Y N, Xi J M, et al. Petal effect: A superhydrophobic state with high adhesive force. Langmuir, 2008, 24: 4114-4119

10 Nosonovsky M. Multiscale roughness and stability of superhydrophobic biomimetic interfaces. Langmuir, 2007, 23: 3157-3161

11 Autumn K, Liang Y A, Hsieh S T, et al. Adhesive force of a single gecko foot-hair. Nature, 2000, 405: 681-685

12 Barthlott W, Neinhuis C. Purity of the sacred lotus, or escape from contamination in biological surfaces. Planta, 1997, 202: 1-8

13 Gu Z Z, Uetsuka H, Takahashi K, et al. Structural color and the lotus effect. Angew Chem Int Ed, 2003, 42: 894-897

14 Ball P. Engineering shark skin and other solutions. Nature, 1999, 400: 507-509

15 Feng X J, Jiang L. Design and creation of superwetting/antiwetting surfaces. Adv Mater, 2006, 18: 3063-3078

16 Feng L, Li S H, Li Y S, et al. Super-hydrophobic surfaces: From natural to artificial. Adv Mater, 2002, 14: 1857-1860

17 Cao L L, Hu H H, Gao D. Design and fabrication of micro-textures for inducing a superhydrophobic behavior on hydrophilic materials. Langmuir, 2007, 23: 4310-4314

18 Lee W, Jin M K, Yoo W C, et al. Nanostructuring of a polymeric substrate with well-defined nanometer-scale topography and tailored surface wettability. Langmuir, 2004, 20: 7665-7669

19 Wang Y, Mo Y F, Zhu M, et al. Wettability of metal coatings with biomimic micro textures. Surf Coat Technol, 2008, 203: 137-141

$20 \mathrm{Xu} \mathrm{Y} \mathrm{X,} \mathrm{Bai} \mathrm{H,} \mathrm{Lu} \mathrm{G} \mathrm{W,} \mathrm{et} \mathrm{al.} \mathrm{Flexible} \mathrm{graphene} \mathrm{films} \mathrm{via} \mathrm{the} \mathrm{filtra-}$ tion of water-soluble noncovalent functionalized graphene sheets. J Am Chem Soc, 2008, 130: 5856-5857

21 Choucair M, Thordarson P, Stride J A. Gram-scale production of graphene based on solvothermal synthesis and sonication. Nat Nanotechnol, 2009, 4: 30-33

22 Yan X B, Chen J T, Yang J, et al. Fabrication of free-standing, electrochemically active, and biocompatible graphene oxide-polyaniline and graphene-polyaniline hybrid papers. ACS Appl Mater Interfaces, 2010, 2: 2521-2529

23 Stankovich S, Dikin D A, Piner R D, et al. Synthesis of graphene-based nanosheets via chemical reduction of exfoliated graphite oxide. Carbon, 2007, 45: 1558-1565

24 Ramesha G K, Sampath S. Electrochemical reduction of oriented graphene oxide films: An in situ Raman spectroelectrochemical study. J Phy Chem C, 2009, 19: 7985-7989

Open Access This article is distributed under the terms of the Creative Commons Attribution License which permits any use, distribution, and reproduction in any medium, provided the original author(s) and source are credited.

\section{Supporting Information}

SI 1 Preparation of graphene oxide (GO)

SI 2 Structural characterization of GO and GNS

Figure S1 Characterization of GO: XRD patterns (a) and TEM image (b).

Figure S2 The C 1s XPS spectra of the textured GO film and the textured graphene film. It is revealed that the GO film was deoxygenized by electrochemical reduction.

The supporting information is available online at csb.scichina.com and www.springerlink.com. The supporting materials are published as submitted, without typesetting or editing. The responsibility for scientific accuracy and content remains entirely with the authors. 